\title{
Prevalência e intervenção dos riscos ocupacionais no processo de trabalho dos enfermeiros: revisão integrativa da literatura
}

\author{
Patrick Leonardo Nogueira da Silva \\ Acadêmico do curso de graduação em Medicina pelas Faculdades Unidas do Norte de Minas - FUNORTE \\ Enfermeiro especialista em Saúde da Família e Enfermagem do Trabalho \\ Mestrando em Cuidados Primários em Saúde pela Universidade Estadual de Montes Claros - UNIMONTES \\ $\triangle$ patrick_mocesp70@hotmail.com
}

\author{
Álvaro Ataíde Landulfo Teixeira \\ Enfermeiro pelas Faculdades Integradas Pitágoras de Montes Claros - FIPMOC
}

Ana Izabel de Oliveira Neta

Professora do curso de graduação em Enfermagem das Faculdades Santo Agostinho de Montes Claros - FASA Enfermeira especialista em Saúde Coletiva com ênfase em Saúde da Família e Gestão Microrregional em Saúde

\author{
Valdira Vieira de Oliveira \\ Professora do curso de graduação em Enfermagem das Faculdades Santo Agostinho de Montes Claros - FASA \\ Enfermeira especialista em Enfermagem em Saúde Pública \\ Mestrado em Enfermagem pela Universidade Federal de São Paulo - UNIFESP

\section{Aurelina Gomes e Martins} \\ Professora do curso de graduação em Enfermagem da Universidade Estadual de Montes Claros - UNIMONTES \\ Enfermeira especialista em Enfermagem de Saúde Pública \\ Mestrado em Saúde Pública pela Universidade Federal de São Paulo - UNIFESP

\section{Elaine Cristina Santos Alves} \\ Professora do curso de graduação em Enfermagem da Universidade Estadual de Montes Claros - UNIMONTES \\ Enfermeira especialista em Vigilância Sanitária e Epidemiológica \\ Mestrado em Ciências da Saúde pela Universidade Federal de São Paulo - UNIFESP

\section{Maricy Kariny Soares Oliveira} \\ Professora do curso de graduação em Enfermagem da Universidade Estadual de Montes Claros - UNIMONTES \\ Enfermeira especialista em Auditoria em Sistemas de Saúde \\ Doutorado em Ciências da Educação pela Universidade Evangelista do Paraguai e Cambridge - UEP

\section{Carolina dos Reis Alves} \\ Professora do curso de graduação em Enfermagem da Universidade Estadual de Montes Claros - UNIMONTES \\ Enfermeira especialista em Saúde da Família \\ Doutorado em Ciências da Saúde pela Universidade Estadual de Montes Claros - UNIMONTES
}

\section{Resumo:}

O acidente de trabalho ocorre durante o exercício laboral dentro da empresa. Os trabalhadores de enfermagem expõem-se a diversos riscos ocupacionais, de modo a evidenciar prevalência dos riscos biológicos. Objetivou-se investigar a prevalência, bem como a intervenção, dos riscos ocupacionais no processo de trabalho dos enfermeiros. Trata-se de um estudo descritivo, exploratório, com suporte em uma revisão integrativa da literatura, realizada nas seguintes bases de dados: Literatura LatinoAmericana e do Caribe em Ciências da Saúde e Scientific Electronic Library Online. A coleta de dados foi realizada durante o $2^{\circ}$ semestre de 2016. A amostra foi composta por 30 artigos científicos publicados durante o período de 2009-2016. Na amostra analisada, houve prevalência dos acidentes de trabalho na equipe de enfermagem decorrente da manipulação de material perfurocortante contaminado com fluido biológico. A categoria mais acometida foram os técnicos de enfermagem. Nestes estudos, a sobrecarga de trabalho associado ao estresse físico e psíquico é apontada como a principal causa destes acidentes. Fatores de 
risco físico, químico ou de acidente apresentam contribuição mínima para a sua ocorrência. É percebido que em alguns estudos, os equipamentos de proteção individual não são devidamente disponibilizados ao trabalhador de modo a facilitar a aquisição de um acidente de trabalho, bem como uma doença osteomuscular relacionada ao trabalho. Portanto, a criação de protocolos e programas de educação permanente para os profissionais de saúde é de fundamental importância para o aperfeiçoamento quanto às formas práticas e rotineiras de prevenção dos acidentes, bem como da exposição às situações de risco. Palavras-chave: Saúde do trabalhador, Riscos ocupacionais, Prevalência.

\title{
Prevalence and intervention of occupational risks in the work process of nurses: integrative literature review
}

\begin{abstract}
:
The work accident occurs during the work exercise within the company. Nursing workers are exposed to various occupational hazards in order to highlight the prevalence of biological risks. The objective was to investigate the prevalence, as well as the intervention, of occupational risks in the work process of nurses. This is a descriptive, exploratory study, supported by an integrative literature review, conducted in the following databases: Latin American and Caribbean Health Sciences Literature and Scientific Electronic Library Online. Data collection was performed during the second semester of 2016. The sample consisted of 30 scientific articles published during the period 2009-2016. In the sample analyzed, there was a prevalence of occupational accidents in the nursing staff due to the handling of sharps contaminated with biological fluid. The most affected category were nursing technicians. In these studies, work overload associated with physical and mental stress is pointed as the main cause of these accidents. Physical, chemical or accident risk factors make minimal contribution to their occurrence. It is noted that in some studies, personal protective equipment is not properly available to the worker in order to facilitate the acquisition of a work accident, as well as a work-related musculoskeletal disease. Therefore, the creation of protocols and programs of permanent education for health professionals is of fundamental importance for the improvement of practical and routine forms of accident prevention, as well as exposure to risk situations.
\end{abstract}

Keywords: Worker health, Occupational risks, Prevalence.

\section{Prevalencia e intervención de riesgos laborales en el proceso de trabajo de las enfermeras: revisión integradora de literatura}

\section{Resumen:}

El accidente laboral ocurre durante el ejercicio laboral dentro de la empresa. Los trabajadores de enfermería están expuestos a diversos riesgos laborales para resaltar la prevalencia de riesgos biológicos. El objetivo fue investigar la prevalencia, así como la intervención, de los riesgos laborales en el proceso de trabajo de las enfermeras. Este es un estudio descriptivo, exploratorio, respaldado por una revisión bibliográfica integradora, realizada en las siguientes bases de datos: Literatura y Biblioteca Científica Electrónica de Ciencias de la Salud de América Latina y el Caribe en línea. La recolección de datos se realizó durante el segundo semestre de 2016. La muestra consistió en 30 artículos científicos publicados durante el período 2009-2016. En la muestra analizada, hubo una prevalencia de accidentes laborales en el personal de enfermería debido al manejo de objetos punzantes contaminados con fluido biológico. La categoría más afectada fueron los técnicos de enfermería. En estos estudios, la sobrecarga de trabajo asociada con el estrés físico y mental se señala como la principal causa de estos accidentes. Los factores de riesgo físicos, químicos o de accidentes contribuyen mínimamente a su aparición. Se observa que, en algunos estudios, el equipo de protección personal no está disponible adecuadamente para el trabajador a fin de facilitar la adquisición de un accidente laboral, así como una enfermedad musculoesquelética relacionada con el trabajo. Por lo tanto, la creación de protocolos y programas de educación permanente para profesionales de la salud es de fundamental importancia para la mejora de las formas prácticas y rutinarias de prevención de accidentes, así como la exposición a situaciones de riesgo.

Palabras clave: Salud laboral, Riesgos laborales, Prevalencia. 


\section{INTRODUÇÃO}

$\mathrm{O}$ acidente de trabalho (AT) é aquele que ocorre pelo exercício do trabalho ou a serviço da empresa. Os trabalhadores de enfermagem estão expostos aos diversos riscos ocupacionais, estando bem evidentes os riscos biológicos, seguidos dos químicos, dos psicossociais e, em menor escala, dos riscos físicos, de acidentes e ergonômicos (AREOSA, 2011). Os danos ao trabalhador podem ser devido às condições deficientes de trabalho, como as lesões por AT, as doenças ocupacionais, a fadiga, a insatisfação, o estresse e as patologias inespecíficas. Além disso, vale ressaltar que a equipe de enfermagem que atua em instituições hospitalares, com déficits de recursos humanos e materiais, convive com um trabalho mais penoso, o que provoca agravos à saúde do trabalhador (ST) (DUARTE; MAURO, 2010).

Embora os profissionais se sintam satisfeitos com o trabalho, eles reconhecem que o ambiente os expõe a alguma insatisfação relacionada às relações interpessoais, o que configura exposição a riscos psicossociais, os quais podem causar prejuízo à saúde física e mental. Esses riscos permitem uma associação com os aspectos subjetivos a qual reflete a história pessoal de cada enfermeiro (ESPINDOLA; FONTANA, 2012). Os fatores de riscos ambientais no trabalho de enfermagem encontram-se presentes diariamente nas suas atividades, cabendo aos gestores fazer uma avaliação dos mesmos de forma a minimizarem os seus impactos sobre a vida desses profissionais. É importante desencadear um processo de treinamento eficaz e uma avaliação do impacto, junto aos trabalhadores (DUARTE; MAURO, 2010).

São necessárias ações de promoção e prevenção à vida pessoal e individual, externa à sua atividade laboral, que promovam educação em saúde também fora do trabalho. Os profissionais devem fazer o uso correto e rotineiro de equipamentos de proteção individual (EPI) e observar os cuidados para evitar os AT (NASCIMENTO; VIEIRA; CUNHA, 2010). Diante da inegável importância do assunto em discussão, este estudo propõe levantar as principais publicações a respeito dos riscos ocupacionais a que os enfermeiros estão expostos rotineiramente. Pois sem dúvida, o tema proposto é importante para nós profissionais da enfermagem e de um modo geral contribui para o aumento do conhecimento e desta forma minimizar os riscos expostos ao enfermeiro no ambiente de trabalho. 
Sendo assim, este estudo objetivou investigar a prevalência e intervenções dos riscos ocupacionais no processo de trabalho da equipe de enfermagem por meio de uma revisão integrativa da literatura científica.

\section{MÉTODO}

Trata-se de um estudo descritivo, exploratório, com suporte em uma revisão integrativa da literatura. A pesquisa procedeu conforme as seguintes etapas: elaboração da pergunta norteadora; busca ou amostragem na literatura; coleta de dados; e análise crítica dos estudos incluídos.

Este estudo foi realizado nos bancos de dados indexados da Biblioteca Virtual de Saúde (BVS), sendo estes: Literatura Latino-Americana e do Caribe em Ciências da Saúde (LILACS) e Scientific Electronic Library Online (SciELO). A busca consistia na seleção de artigos científicos publicados durante o período de 2009-2016. Este período foi escolhido com o objetivo de obter publicações mais recentes relacionadas ao tema. A coleta de dados foi realizada durante o $2^{\circ}$ semestre de 2016. Foram utilizados os seguintes descritores para captação da amostra: “enfermagem", "riscos ocupacionais", "saúde do trabalhador".

Foram encontradas 96 publicações. Inicialmente realizou-se a leitura dos títulos e resumos para análise das publicações, foram utilizados os seguintes critérios de inclusão: artigos de periódicos nacionais e internacionais indexados e publicados durante o período de 2009-2016; apresentar resumos disponibilizados em língua portuguesa; artigos escritos na língua portuguesa e relacionados ao tema na qual se encontram disponíveis gratuitamente online para download. As publicações que não disponibilizaram o texto completo foram excluídas do estudo. A amostra final do estudo foi constituída de 30 publicações.

Após o levantamento de dados, foi realizada a leitura dos resumos para inclusão e as informações obtidas relacionadas aos artigos foram organizadas em um quadro sinóptico, posteriormente comparadas e analisadas entre si, proporcionando conhecer nitidamente a produção científica relacionada aosriscos ocupacionais na enfermagem.

As pesquisas selecionadas foram resumidas e transcritas no quadro sinóptico com as variáveis analisadas. 0 presente estudo foi delineado obedecendo às etapas metodológicas acima descritas. A análise do material foi realizada através de leitura crítica na qual permitiu identificar convergências e possibilitaro seguinte agrupamento por categorias temáticas: "Abordagem dos tipos de riscos ocupacionais geradores de AT na rotina da equipe de 
enfermagem", "Expandindo o conhecimento sobre os riscos ocupacionais e os AT: exposição dos profissionais e os procedimentos realizados", "Prevenção dos AT nos profissionais de enfermagem: definindo e discutindo as intervenções dentro do processo de trabalho".

\section{RESULTADOS}

Observa-se que, do total amostral selecionado, 16 artigos são provenientes da base de dados SciELO e 14 artigos advém do LILACS (Tabela 1). Quanto ao ano de publicação dos estudos selecionados, houve maior publicação em 2012 (26,6\%). Quanto à metodologia das publicações utilizadas pelos autores dos estudos selecionados, predominou o tipo "descritivo exploratório" (40\%). Verificou-se a maior quantidade de trabalhos publicados pelos periódicos: Rev Bras Enferm e Acta Paul Enferm (13,5\%). As demais obtiveram um menor número de publicações (Tabela 2).

Tabela 1 - Distribuição dos artigos segundo os descritores utilizados e as bases de dados

\begin{tabular}{|c|c|c|c|c|}
\hline Base de dados & Descritores & AS & $\mathrm{AE}$ & $\overline{A U}$ \\
\hline SCiELO & $\begin{array}{l}\text { Enfermagem } \\
\text { Riscos ocupacionais } \\
\text { ST }\end{array}$ & 56 & 40 & 16 \\
\hline LILACS & $\begin{array}{l}\text { Enfermagem } \\
\text { Riscos ocupacionais } \\
\text { ST }\end{array}$ & 40 & 26 & 14 \\
\hline Total & & 96 & 66 & 30 \\
\hline
\end{tabular}


Tabela 2 - Distribuição amostral conforme a base de dados, ano, método e periódico. $(n=30)$

\begin{tabular}{|c|c|c|c|}
\hline Variáveis & Descrição & $\mathbf{n}$ & $\%$ \\
\hline \multirow{2}{*}{ Base de dados } & SciELO & 16 & 53,3 \\
\hline & LILACS & 14 & 46,7 \\
\hline \multirow{8}{*}{ Ano } & 2009 & 04 & 13,6 \\
\hline & 2010 & 05 & 16,5 \\
\hline & 2011 & 06 & 20,0 \\
\hline & 2012 & 08 & 26,6 \\
\hline & 2013 & 06 & 20,0 \\
\hline & 2014 & 01 & 3,3 \\
\hline & 2015 & 00 & 00 \\
\hline & 2016 & 00 & 00 \\
\hline \multirow{6}{*}{ Método } & Transversal & 07 & 23,1 \\
\hline & Descritivo exploratório & 12 & 40,0 \\
\hline & Revisão integrativa & 01 & 3,3 \\
\hline & Descritivo retrospectivo & 03 & 10,0 \\
\hline & Revisão sistemática & 03 & 10,0 \\
\hline & Observacional & 04 & 13,6 \\
\hline \multirow{17}{*}{ Periódico } & Rev Bras Enferm & 04 & 13,6 \\
\hline & Acta Paul Enferm & 04 & 13,6 \\
\hline & Esc Anna Nery & 03 & 10,0 \\
\hline & Rev Pesq Cuid Fundam & 03 & 10,0 \\
\hline & Rev Gaúcha Enferm & 02 & 6,6 \\
\hline & Rev Esc Enferm USP & 02 & 6,6 \\
\hline & Rev Min Enferm & 02 & 6,6 \\
\hline & Ciênc Cuid Saúde & 01 & 3,3 \\
\hline & J Bras Enferm & 01 & 3,3 \\
\hline & Texto Contexto - Enferm & 01 & 3,3 \\
\hline & Rev Saúde Pública & 01 & 3,3 \\
\hline & Rev Enferm UERJ & 01 & 3,3 \\
\hline & Rev CEFAC & 01 & 3,3 \\
\hline & Ciênc Enferm & 01 & 3,3 \\
\hline & Rev Bras Saúde Ocup & 01 & 3,3 \\
\hline & Rev Enferm UFPE On Line & 01 & 3,3 \\
\hline & Rev Eletr Enferm & 01 & 3,3 \\
\hline
\end{tabular}

Fonte: BVS, 2016.

A Tabela 3 aborda o resumo de toda a amostra (A1-A30) do estudo de acordo a sua codificação, título, autor, método e nível de evidencia (NE). O Quadro 1 complementa os achados do estudo com a síntese dos resultados da amostra captada. 
Tabela 3 - Distribuição da amostra do estudo com relação ao código, título, autor, método e NE.

\begin{tabular}{|c|c|c|c|c|}
\hline Código & Título & Autor & Método & $\mathrm{NE}$ \\
\hline A1 & $\begin{array}{l}\text { Organização do trabalho de enfermagem na Atenção Básica (AB): } \\
\text { uma questão para a saúde do trabalhador. }\end{array}$ & David et al. (2009) & $\begin{array}{l}\text { Estudo transversal, descritivo e } \\
\text { inferencial. }\end{array}$ & 1B \\
\hline A2 & $\begin{array}{l}\text { Os sentimentos vivenciados após exposição ocupacional entre } \\
\text { trabalhadores de saúde: fulcro para repensar o trabalho em } \\
\text { instituições de saúde. }\end{array}$ & Sarquis e Felli (2009) & $\begin{array}{l}\text { Utilização de grupo focal com abordagem } \\
\text { qualitativa. }\end{array}$ & $2 \mathrm{C}$ \\
\hline A3 & $\begin{array}{l}\text { Problemas de saúde de trabalhadores de enfermagem em } \\
\text { ambulatórios pela exposição às cargas fisiológicas. }\end{array}$ & Sápia, Felli e Ciampone (2009) & $\begin{array}{l}\text { Estudo descritivo com abordagem quanti- } \\
\text { qualitativa. }\end{array}$ & $2 \mathrm{C}$ \\
\hline A4 & Investigação de acidentes biológicos entre profissionais de saúde. & Silva et al. (2009) & $\begin{array}{l}\text { Pesquisa descritiva, documental, com } \\
\text { abordagem quantitativa. }\end{array}$ & $2 \mathrm{C}$ \\
\hline A5 & $\begin{array}{l}\text { Risco de infecção tuberculosa em agentes comunitários de saúde } \\
\text { (ACS). }\end{array}$ & Moreira, Zandonade e Maciel (2010) & $\begin{array}{l}\text { Estudo de coorte, prospectivo, com } \\
\text { abordagem quantitativa. }\end{array}$ & $2 \mathrm{~B}$ \\
\hline A6 & $\begin{array}{l}\text { Condições de saúde autorreferidas de professores de uma } \\
\text { universidade regional. }\end{array}$ & Fontana e Pinheiro (2010) & $\begin{array}{l}\text { Estudo epidemiológico, transversal, com } \\
\text { abordagem quantitativa. }\end{array}$ & $1 \mathrm{~B}$ \\
\hline A7 & Influência da exposição a material biológico na adesão ao uso de EPI. & Ribeiro et al. (2010) & $\begin{array}{l}\text { Estudo descritivo, com abordagem } \\
\text { qualitativa. }\end{array}$ & $2 \mathrm{C}$ \\
\hline A8 & $\begin{array}{l}\text { Rede de Prevenção de Acidentes de Trabalho: uma estratégia de } \\
\text { ensino a distância. }\end{array}$ & Marziale et al. (2010) & $\begin{array}{l}\text { Estudo quase experimental baseado no } \\
\text { Modelo de Promoção da Saúde de Pender. }\end{array}$ & $2 \mathrm{C}$ \\
\hline A9 & $\begin{array}{l}\text { Condições de trabalho da enfermagem nas enfermarias de um } \\
\text { hospital universitário. }\end{array}$ & Mauro et al. (2010) & $\begin{array}{l}\text { Estudo não experimental, com abordagem } \\
\text { quantitativa. }\end{array}$ & $2 \mathrm{C}$ \\
\hline A10 & $\begin{array}{l}\text { Uma contribuição à saúde dos trabalhadores: um guia sobre } \\
\text { exposição aos fluídos biológicos. }\end{array}$ & Miranda et al. (2011) & $\begin{array}{l}\text { Construção de um guia que contribua para } \\
\text { a adesão ao protocolo de monitoramento } \\
\text { biológico }\end{array}$ & $2 \mathrm{~A}$ \\
\hline A11 & $\begin{array}{l}\text { Investigação de acidentes biológicos entre profissionais da equipe } \\
\text { multidisciplinar de um hospital. }\end{array}$ & Câmara et al. (2011) & $\begin{array}{l}\text { Estudo descritivo, documental, com } \\
\text { abordagem quantitativa. }\end{array}$ & $2 \mathrm{C}$ \\
\hline A12 & $\begin{array}{l}\text { Ruído no contexto hospitalar: impacto na saúde dos profissionais de } \\
\text { enfermagem. }\end{array}$ & Costa (2011) & $\begin{array}{l}\text { Estudo descritivo, transversal, com } \\
\text { abordagem quantitativa. }\end{array}$ & $1 \mathrm{~B}$ \\
\hline A13 & $\begin{array}{l}\text { Acidentes com material biológico em trabalhadores de enfermagem } \\
\text { do Hospital Geral de Palmas (TO). }\end{array}$ & Machado e Machado (2011) & $\begin{array}{l}\text { Estudo descritivo, transversal, com } \\
\text { abordagem quantitativa. }\end{array}$ & 1B \\
\hline A14 & $\begin{array}{l}\text { Percepção de técnicos de enfermagem sobre o uso de EPI em um } \\
\text { serviço de urgência. }\end{array}$ & Guimarães et al. (2011) & $\begin{array}{l}\text { Estudo descritivo, com abordagem } \\
\text { qualitativa. }\end{array}$ & $2 \mathrm{C}$ \\
\hline A15 & $\begin{array}{l}\text { Exposição ocupacional por material biológico no Hospital Santa Casa } \\
\text { de Pelotas - } 2004 \text { a } 2008 \text {. }\end{array}$ & Lima, Oliveira e Rodrigues (2011) & $\begin{array}{l}\text { Estudo descritivo, transversal, com } \\
\text { abordagem quantitativa. }\end{array}$ & $1 \mathrm{~B}$ \\
\hline
\end{tabular}

Revista SUSTINERE, Rio de Janeiro, v. 9, n.2, p. 463-477, jul-dez, 2021 


\begin{tabular}{|c|c|c|c|c|}
\hline A16 & $\begin{array}{l}\text { As crenças dos trabalhadores de saúde nos AT com exposição a fluido } \\
\text { biológico: pesquisa descritiva. }\end{array}$ & Wall et al. (2011) & $\begin{array}{l}\text { Estudo descritivo, com abordagem quanti- } \\
\text { qualitativa. }\end{array}$ & $2 \mathrm{C}$ \\
\hline A17 & $\begin{array}{l}\text { Internação domiciliar: risco de exposição biológica para a equipe de } \\
\text { saúde. }\end{array}$ & Figueiredo e Maroldi (2012) & $\begin{array}{l}\text { Estudo exploratório, prospectivo, com } \\
\text { abordagem quantitativa. }\end{array}$ & $2 \mathrm{~B}$ \\
\hline A18 & $\begin{array}{l}\text { Adesão às precauções-padrão de profissionais de enfermagem de um } \\
\text { hospital universitário. }\end{array}$ & Malaguti-Toffano et al. (2012) & $\begin{array}{l}\text { Estudo transversal, comparativo, com } \\
\text { abordagem quantitativa. }\end{array}$ & $1 \mathrm{~B}$ \\
\hline A19 & $\begin{array}{l}\text { Acidentes ocupacionais com materiais perfurocortantes em hospitais } \\
\text { de Montes Claros-MG. }\end{array}$ & Ruas et al. (2012) & $\begin{array}{l}\text { Estudo descritivo, retrospectivo, com } \\
\text { abordagem quantitativa. }\end{array}$ & $2 \mathrm{C}$ \\
\hline A20 & $\begin{array}{l}\text { Fatores estressores para a equipe de enfermagem da unidade de } \\
\text { terapia intensiva (UTI). }\end{array}$ & Rodrigues (2012) & Revisão da literatura. & $1 \mathrm{~A}$ \\
\hline A21 & Risco e vulnerabilidade nas práticas dos profissionais de saúde. & Santos et al. (2012) & Revisão da literatura. & $1 \mathrm{~A}$ \\
\hline A22 & Riscos ocupacionais no processamento de sistemas de hemodiálise. & Hoefel, Lautert e Fortes (2012) & $\begin{array}{l}\text { Estudo observacional, exploratório, com } \\
\text { abordagem quantitativa. }\end{array}$ & $2 \mathrm{~B}$ \\
\hline A23 & $\begin{array}{l}\text { Riscos ocupacionais e mecanismos de autocuidado do trabalhador de } \\
\text { um centro de material e esterilização. }\end{array}$ & Espindola e Fontana (2010) & $\begin{array}{l}\text { Estudo descritivo, com abordagem } \\
\text { qualitativa. }\end{array}$ & $2 \mathrm{C}$ \\
\hline A24 & $\begin{array}{l}\text { O conceito de risco e os seus efeitos simbólicos nos acidentes com } \\
\text { instrumentos perfuro cortantes. }\end{array}$ & Silva, Lima e Marziale (2012) & $\begin{array}{l}\text { Estudo descritivo, exploratório, com } \\
\text { abordagem qualitativa. }\end{array}$ & $2 \mathrm{~B}$ \\
\hline A25 & $\begin{array}{l}\text { Enfermagem e trabalho: fundamentos para a atenção à saúde dos } \\
\text { trabalhadores. }\end{array}$ & Moreira et al. (2013) & Resenha da literatura científica. & $1 \mathrm{~A}$ \\
\hline A26 & $\begin{array}{l}\text { Concepções da equipe de enfermagem sobre a exposição a riscos } \\
\text { físicos e químicos no ambiente hospitalar. }\end{array}$ & Sulzbacher e Fontana (2013) & $\begin{array}{l}\text { Estudo descritivo, exploratório, com } \\
\text { abordagem quantitativa. }\end{array}$ & $2 \mathrm{~B}$ \\
\hline A27 & A produção científica sobre a saúde do trabalhador de enfermagem. & Valença et al. (2013) & Revisão sistemática da literatura. & $1 \mathrm{~A}$ \\
\hline A28 & $\begin{array}{l}\text { A biossegurança durante os cuidados de enfermagem aos pacientes } \\
\text { com tuberculose pulmonar. }\end{array}$ & Costa et al. (2013) & $\begin{array}{l}\text { Estudo descritivo, } \\
\text { retrospectivo, comploratório, } \\
\text { quantitativa. }\end{array}$ & $2 \mathrm{~B}$ \\
\hline A29 & $\begin{array}{l}\text { Riscos e doenças ocupacionais do docente universitário de } \\
\text { enfermagem: implicações na saúde do trabalhador. }\end{array}$ & Oliveira et al. (2013) & $\begin{array}{l}\text { Estudo de campo, descritivo, exploratório, } \\
\text { com abordagem quanti-qualitativa. }\end{array}$ & $2 \mathrm{~B}$ \\
\hline A30 & $\begin{array}{l}\text { O enfermeiro do trabalho na identificação dos riscos ocupacionais em } \\
\text { medicina hiperbárica. }\end{array}$ & Barboza, Cortez e Valente (2014) & $\begin{array}{l}\text { Estudo descritivo, exploratório, com } \\
\text { abordagem qualitativa. }\end{array}$ & $2 \mathrm{~B}$ \\
\hline
\end{tabular}


Quadro 1 - Distribuição da amostra do estudo conforme síntese dos resultados.

\begin{tabular}{|c|c|}
\hline Código & Síntese dos resultados \\
\hline A1 & $\begin{array}{l}\text { Associação estatisticamente significativa foi encontrada entre as variáveis "clima } \\
\text { ruim de trabalho com os companheiros" e "conflito com clientes", "má } \\
\text { organização de turnos e horários" e "ritmo acelerado". }\end{array}$ \\
\hline A2 & $\begin{array}{l}\text { O medo foi o primeiro sentimento expresso. O medo que o trabalhador sofre no } \\
\text { desempenho de suas atribuições, potencia o desenvolvimento de situações } \\
\text { estressantes geradas no ambiente de trabalho e desencadeiam sofrimento } \\
\text { psíquico. Os sentimentos de raiva e revolta aparecem nos discursos diante da } \\
\text { indignação do trabalhador. Outro sentimento manifestado pelos sujeitos é a culpa } \\
\text { pelo desencadeamento do AT. }\end{array}$ \\
\hline A3 & $\begin{array}{l}\text { A alta frequência de exposição dos trabalhadores a cargas fisiológicas (42,1\%), } \\
\text { associada ao alto índice de massa corporal (IMC) ( } 26,3 \% \text { obesos) é geradora de } \\
\text { distúrbios osteomusculares relacionados à prática do trabalho (DORT), cujo } \\
\text { principal sintoma é a dor em diferentes regiões. Também as varizes e os } \\
\text { microvasos nas pernas e calosidades nos pés são relatados. }\end{array}$ \\
\hline A4 & $\begin{array}{l}\text { Observou-se que a categoria profissional mais exposta foi a dos auxiliares e } \\
\text { técnicos de enfermagem }(54,1 \%) \text {, seguida pela dos acadêmicos de medicina e } \\
\text { odontologia (10,4\%). }\end{array}$ \\
\hline A5 & $\begin{array}{l}\text { A incidência da viragem foi de } 41,7 \% \text { no grupo dos expostos e } 13,5 \% \text { no grupo dos } \\
\text { não expostos. O risco anual de infecção foi de } 52,8 \% \text { no grupo dos expostos e de } \\
14,4 \% \text { no grupo dos não expostos ( } p=0,013) \text {. Observou-se associação entre } \\
\text { viragem tuberculínica e exposição à paciente com tuberculose (TBC) (RR=3,08; } \\
\text { IC95\%: } 1,201 ; 7,914) \text {. }\end{array}$ \\
\hline A6 & $\begin{array}{l}\text { A lombalgia e o cansaço foram queixas prevalentes e a exposição aos riscos } \\
\text { biológicos são frequentes na atividade ocupacional dos respondentes. A } \\
\text { aprendizagem dos alunos representa a maior satisfação no trabalho. }\end{array}$ \\
\hline A7 & $\begin{array}{l}\text { Observou-se que a experiência desta exposição influencia em maior ou menor } \\
\text { escala na mudança de comportamento para a adesão ao EPI. Contudo, esse } \\
\text { comportamento é individual e sofre influências de outros fatores, como ausência } \\
\text { ou inadequação do EPI, sobrecarga de trabalho, pressa e situações de emergência. }\end{array}$ \\
\hline A8 & $\begin{array}{l}\text { Antes do treinamento 58,3\% dos trabalhadores usavam luvas para administrar } \\
\text { medicamentos endovenosos na semana que antecedeu o treinamento e } 83,3 \% \\
\text { trabalhadores informaram intenção do uso de luvas após o treinamento. }\end{array}$ \\
\hline A9 & Foram indicados os fatores de riscos biológicos, físicos, ergonômicos e químicos. \\
\hline A10 & $\begin{array}{l}\text { A exposição ocupacional entre estes, mais especificamente, entre os da } \\
\text { enfermagem, pode ser atribuída a vários fatores direta ou indiretamente, tais } \\
\text { como: a assistência integral e direta ao paciente, administrando medicação e } \\
\text { realizando curativos; limpeza e esterilização de materiais cirúrgicos e } \\
\text { instrumentais diversos, sobrecarga de trabalho; condições impróprias da } \\
\text { realização do processo de trabalho; a não adoção das medidas preventivas, entre } \\
\text { elas o uso dos EPI, e a não vacinação contra hepatite B; entre outros. }\end{array}$ \\
\hline A11 & $\begin{array}{l}\text { A categoria profissional mais exposta foi a dos técnicos de enfermagem (50\%), } \\
\text { seguida dos auxiliares de enfermagem }(25,53 \%) \text {, e a principal causa de ocorrência } \\
\text { foi devida ao descarte inadequado de material perfurocortante }(43,62 \%) \text {. }\end{array}$ \\
\hline A12 & $\begin{array}{l}\text { As principais fontes de ruído citadas foram equipamentos com alarmes (36,23\%); } \\
\text { conversas altas e risadas }(34,06 \%) \text {; e movimentação de pessoas }(18,12 \%) \text {. A queixa }\end{array}$ \\
\hline
\end{tabular}




\begin{tabular}{|c|c|}
\hline & $\begin{array}{l}\text { auditiva mais citada foi o zumbido (14,49\%), e as extra-auditivas foram à irritação } \\
\text { (45,63\%); seguida pela alteração do sono e dor de cabeça }(44,20 \%) \text {; e baixa } \\
\text { concentração }(34,78 \%) \text {. }\end{array}$ \\
\hline A13 & $\begin{array}{l}\text { Dentre os profissionais de enfermagem, } 178(45,7 \%) \text { declararam já ter sofrido AT } \\
\text { com material biológico ( } 55,6 \% \text { por perfurocortante e } 44,4 \% \text { com fluidos), sendo } \\
106(59,9 \%) \text { técnicos de enfermagem, } 57(32 \%) \text { auxiliares de enfermagem e } 15 \\
(8,4 \%) \text { enfermeiros. }\end{array}$ \\
\hline A14 & $\begin{array}{l}\text { Os técnicos de enfermagem demonstraram-se inseguros na compreensão sobre } \\
\text { os riscos biológicos ocupacionais durante o atendimento, no desconhecimento } \\
\text { sobre as Normas Regulamentadoras (NR) nove e } 32 \text { e sobre a utilização adequada } \\
\text { dos EPI no atendimento às vítimas, além do não fornecimento regular destes pela } \\
\text { instituição. }\end{array}$ \\
\hline A15 & $\begin{array}{l}\text { Como principal resultado, encontrou-se a maior ocorrência de AT com material } \\
\text { biológico entre os profissionais técnicos em enfermagem do sexo feminino } \\
(38,6 \%) \text {, com idade de } 21 \text { a } 30 \text { anos }(53,9 \%) \text {. A maioria dos AT aconteceu através de } \\
\text { lesões com perfurocortantes }(82,2 \%) \text {, sendo } 24,1 \% \text { no Centro Cirúrgico e } 84,5 \% \\
\text { envolvendo sangue. }\end{array}$ \\
\hline A16 & $\begin{array}{l}\text { Os trabalhadores demonstram preocupação em contrair Acquired } \\
\text { Immunodeficiency Syndrome (AIDS) e hepatite; referem agregar comportamentos } \\
\text { preventivos ao risco após o evento; percebe o acompanhamento sorológico como } \\
\text { benefício; reconhecem o AT como gerador de estresse emocional e relatam não } \\
\text { haver investimentos das instituições para promover o conhecimento e evitar AT } \\
\text { com exposição biológica. }\end{array}$ \\
\hline A17 & $\begin{array}{l}\text { No acompanhamento das } 159 \text { visitas, realizadas no período de junho de } 2008 \text { a } \\
\text { janeiro de } 2009 \text {, foram observados } 347 \text { procedimentos sendo que, entre os com } \\
\text { risco de exposição biológica, foram identificados curativos (31,1\%), glicemia } \\
\text { capilar (14,4\%) e acesso vascular (3,1\%). A ocorrência de adesão à higienização } \\
\text { prévia das mãos foi de } 21,5 \%, 66,3 \% \text { no uso de luvas e de } 83,5 \% \text { no descarte } \\
\text { adequado do perfurocortante. }\end{array}$ \\
\hline A18 & $\begin{array}{l}\text { Evidenciou-se que } 152(59,4 \%) \text { profissionais apresentaram escores médios altos } \\
\text { para a adesão às precauções padrões (PP), igual ou acima de } 4,5 ; 98(38,3 \%) \text { escores } \\
\text { intermediários, entre } 3,5 \text { e } 4,49 \text { e } 6(2,3 \%) \text { baixos, ou seja, menor que } 3,5 \text {. }\end{array}$ \\
\hline A19 & $\begin{array}{l}\text { Acidentaram-se } 95 \text { profissionais com objetos perfurocortantes envolvendo } \\
\text { material biológico, predominando o sexo feminino }(69,5 \%) \text { e os auxiliares de } \\
\text { enfermagem }(88,4 \%) \text {. O maior índice foi nas enfermarias }(52,6 \%) \text {, com predomínio } \\
\text { das agulhas com lúmen }(87,4 \%) \text { durante o descarte dos objetos }(56,8 \%) \text {. As mãos } \\
\text { foram mais atingidas }(93 \%) \text {. }\end{array}$ \\
\hline A20 & $\begin{array}{l}\text { Revelou-se, neste estudo, a presença de estresse na equipe de enfermagem } \\
\text { atuante na UTI, sendo identificados os principais agentes causadores de estresse. } \\
\text { O estresse está presente no cotidiano dos trabalhadores de enfermagem que } \\
\text { atuam na UTI, podendo desencadear problemas físicos e psíquicos. }\end{array}$ \\
\hline A21 & $\begin{array}{l}\text { Na Atenção Primária à Saúde (APS), os riscos e as vulnerabilidades relacionam-se } \\
\text { à deficiência de recursos para o trabalho, à violência física e ao desgaste } \\
\text { emocional. No contexto hospitalar, destacaram-se os AT com material biológico } \\
\text { relacionado ao uso inadequado e não adesão a medidas de proteção, a sobrecarga } \\
\text { de trabalho e a autoconfiança. }\end{array}$ \\
\hline A22 & $\begin{array}{l}\text { Foram observados } 36 \text { fluxos de reprocessamento compondo } 872 \text { passos com } \\
\text { algum risco potencial. Em } 638(73 \%) \text { observações o risco relacionava-se aos }\end{array}$ \\
\hline
\end{tabular}




\begin{tabular}{|c|c|}
\hline & $\begin{array}{l}\text { pacientes e em 27\%, aos profissionais. Foram observadas técnicas seguras em } 573 \\
\text { (66\%) ocasiões e inseguras em } 299 \text { (34\%). Das } 299 \text { ocasiões inseguras, } 144 \text { (48\%) } \\
\text { dependiam de infraestrutura ou rotina e não do comportamento profissional. }\end{array}$ \\
\hline A23 & $\begin{array}{l}\text { O risco ocupacional mais referido pelos trabalhadores foi o físico, caracterizado } \\
\text { pelo calor. Uso de EPI, hidratação e imunizações são alguns dos mecanismos } \\
\text { utilizados pelos sujeitos para a prevenção de agravos e a promoção de sua saúde. }\end{array}$ \\
\hline A24 & $\begin{array}{l}\text { Os dados foram analisados por meio de uma perspectiva interdisciplinar para } \\
\text { conciliar a análise quantitativa e qualitativa dos dados. }\end{array}$ \\
\hline A25 & $\begin{array}{l}\text { Identifica-se uma linha epistemológica pela evolução histórica e política dos fatos } \\
\text { e as condições enfrentadas pelos trabalhadores no mundo, numa rede de relações } \\
\text { sociais e políticas. }\end{array}$ \\
\hline A26 & $\begin{array}{l}\text { Os trabalhadores perceberam os riscos físicos e químicos a que estão expostos e } \\
\text { referiram reconhecê-los, porém, à análise das respostas, identificou-se } \\
\text { conhecimento deficiente sobre a concepção científica do risco físico. }\end{array}$ \\
\hline A27 & $\begin{array}{l}\text { A maior parte das publicações sobre a ST de enfermagem discute sobre } \\
\text { biossegurança, exposição a riscos biológicos, estresse ocupacional e suas medidas } \\
\text { de controle e prevenção. }\end{array}$ \\
\hline A28 & $\begin{array}{l}\text { O número de casos de TBC no município durante o período do estudo foi de } 65,29 \\
\text { (44,6\%) destes casos foram diagnosticados durante a internação. Quanto às } \\
\text { medidas de biossegurança, durante a admissão dos } 29 \text { casos diagnosticados, foram } \\
\text { encontrados registros de precaução por aerossóis nos prontuários de } 13 \text { ( } 44,8 \%) \\
\text { pacientes. }\end{array}$ \\
\hline A29 & $\begin{array}{l}\text { Condições de trabalho e os riscos do docente; doenças ocupacionais e os motivos } \\
\text { de afastamento; medidas de prevenção. }\end{array}$ \\
\hline A30 & $\begin{array}{l}\text { O trabalho dos profissionais nas clínicas de Medicina Hiperbárica está envolto em } \\
\text { vários fatores de risco ocupacional, destacando-se a probabilidade de incêndio ou } \\
\text { explosão que podem ocasionar danos à ST e até mesmo a morte. O enfermeiro do } \\
\text { trabalho pode atuar orientando/educando os trabalhadores quanto à } \\
\text { importância de utilização de estratégias para eliminar/manterem afastada toda a } \\
\text { fonte de ignição na presença de oxigênio. }\end{array}$ \\
\hline
\end{tabular}

Fonte: Própria.

\section{Prevenção dos AT nos profissionais de enfermagem: intervenções no processo de trabalho}

A maior parte dos autores, além de exporem a respeito do tipo de risco ocupacional, citar os profissionais vulneráveis e discutir os problemas causados pelos AT, também enfocaram a necessidade de programa, de protocolos, reuniões e outras formas de intervir quanto à prevenção dos AT.

Muitos profissionais sabem da importância do uso de EPI, porém não os utilizam com a devida frequência na sua prática laboral devido ao manejo rápido no atendimento, à pressa e à própria falta de hábito de usar EPI, principalmente nos atendimentos de urgência e emergência (SANTOS et al., 2012). É salutar que se deve intervir com estratégias de prevenção 
e promoção de saúde, estimulando a prática de atividades físicas e de lazer, orientações do uso adequado da voz, como também, programar mudanças organizacionais e das condições de trabalho nas instituições de ensino, tendo em vista o que foi evidenciado, visando proporcionar a estes trabalhadores da educação, uma melhoria das condições de saúde (VALENTE et al., 2013).

Os programas de promoção da saúde no local de trabalho destinam-se a aumentar o bem-estar do trabalhador e avançar para um estado de saúde ótimo, bem como reduzir os riscos para a saúde, e devem visar três níveis: conhecimento, alteração comportamental do estilo de vida e ambientes de apoio (MARZIALE et al., 2010). A maior parte das publicações sobre a ST de enfermagem discute sobre biossegurança, exposição a riscos biológicos, estresse ocupacional e suas medidas de controle e prevenção. Ainda há poucos trabalhos publicados acerca da ST de enfermagem na Atenção Básica (AB) ou na Estratégia de Saúde da Família (ESF), sendo a maior parte dos estudos realizados no âmbito hospitalar, principalmente nos hospitais universitários (VALENÇA et al., 2013).

O uso de EPI, hidratação e imunizações são alguns dos mecanismos utilizados pelos sujeitos para a prevenção de agravos e a promoção de sua saúde (ESPINDOLA; FONTANA, 2012). 0 enfermeiro do trabalho pode atuar orientando/educando os trabalhadores quanto à importância de utilização de estratégias para eliminar/manter afastadas todas as fontes de risco (BARBOZA; CORTEZ; VALENTE, 2014). O comportamento para a adesão ao EPI sofre influências de outros fatores, como ausência ou inadequação do EPI, sobrecarga de trabalho, pressa e situações de emergência. A implementação de educação permanente com discussão de situações de risco vivenciadas pelos trabalhadores expostos será necessária para a adesão e uso consciente dos EPI (RIBEIRO et al., 2010).

\section{CONCLUSÃo}

A realização deste trabalho permitiu perceber que todos os profissionais estão expostos aos riscos que o trabalho pode gerar. Mas os profissionais da Enfermagem, devido à maior carga de trabalho ou a exposição constante ao estresse, estão mais vulneráveis ao AT. Existem diversos tipos de riscos, desde a relação do docente no momento da aula, envolvendo sua voz, como também os técnicos que estão na prática diária, e até mesmo os acadêmicos, que estão 
iniciando o contato com o cotidiano. E todos eles levam a não realização do trabalhoefetivo, assim como ao absenteísmo e prejudica a relação interpessoal, tanto de um profissional com o outro, como com o paciente. É realmente necessário instituir protocolos e programas de educação permanente para que os profissionais estejam sempre atentos às formas práticas e rotineiras de prevenir AT e evitarexpor-se à situação de risco.

\section{REFERÊNCIAS}

AREOSA, J. Riscos ocupacionais da imagiologia: estudo de caso num hospital português. Tempo Social. São Paulo, v. 23, n. 2, p. 297-318, 2011. Disponível em: http://dx.doi.org/10.1590/S0103-20702011000200012. Acesso em: 20 ago 2016.

BARBOZA, G. V.; CORTEZ, E. A.; VALENTE, G. S. C. The nurse's work on identification of occupational risks in hyperbaric medicine. Revista de Pesquisa: Cuidado é Fundamental Online. Rio de Janeiro, v. 6, n. 1, p. 320332, 2014. Disponível em: http://dx.doi.org/10.9789/2175-5361.rpcfo.v6.2868. Acesso em: 20 jul 2017.

CÂMARA, P. F.; LIRA, C.; SANTOS JUNIOR, B. J.; VILELLA, T. A. S.; HINRICHSEN, S. L. Investigação de acidentes biológicos entre profissionais da equipe multidisciplinar de um hospital. Revista de Enfermagem UERJ. Rio de Janeiro, v. 19, n. 4, p. 583-586, 2011. Disponível em: http://www.facenf.uerj.br/v19n4/v19n4a13.pdf. Acesso em: 6 dez 2016.

COSTA, G. L. Ruído no contexto hospitalar: impacto na saúde dos profissionais de enfermagem. Revista da Sociedade Brasileira de Fonoaudiologia. São Paulo, v. 16, n. 4, p. 492, 2011. Disponível em: http://dx.doi.org/10.1590/S1516-80342011000400023. Acesso em: 11 dez 2016.

COSTA, M. C. P.; TAMINATO, M.; SILVA, V.; GRANDE, A. J.; BERETTA, A. L. R. Z. A biossegurança durante os cuidados de enfermagem aos pacientes com tuberculose pulmonar. Acta Paulista de Enfermagem. São Paulo, v. 26, n. 4, p. 307-312, 2013. Disponível em: http://dx.doi.org/10.1590/S0103-21002013000400002. Acesso em: 20 jul 2017.

DAVID, H. M. S. L.; MAURO, M. Y. C.; SILVA, V. G.; PINHEIRO, M. A. S.; SILVA, F. H. Organização do trabalho de enfermagem na atenção básica: uma questão para a saúde do trabalhador. Texto \& Contexto - Enfermagem. Florianópolis, v. 18, n. 2, p. 206-214, 2009. Disponível em: http://www.scielo.br/pdf/tce/v18n2/02.pdf. Acesso em: 10 out 2016.

DUARTE, N. S.; MAURO, M. Y. C. Análise dos fatores de riscos ocupacionais do trabalho de enfermagem sob a ótica dos enfermeiros. Revista Brasileira de Saúde Ocupacional. São Paulo, v. 35, n. 121, p. 157-167, 2010. Disponível em: http://dx.doi.org/10.1590/S0303-76572010000100017. Acesso em: 20 ago 2016.

ESPINDOLA, M. C. G.; FONTANA, R. T. Riscos ocupacionais e mecanismos de autocuidado do trabalhador de um centro de material e esterilização. Revista Gaúcha de Enfermagem. Porto Alegre, v. 33, n. 1, p. 116-123, 2012. Disponível em: http://dx.doi.org/10.1590/S1983-14472012000100016. Acesso em: 21 set 2016.

FIGUEIREDO, R. M.; MAROLDI, M. A. C. Internação domiciliar: risco de exposição biológica para a equipe de saúde. Revista da Escola de Enfermagem da USP. São Paulo, v. 46, n. 1, p. 145-150, 2012. Disponível em: http://dx.doi.org/10.1590/S0080-62342012000100020. Acesso em: 19 fev 2017. 
FONTANA, R. T.; PINHEIRO, D. A. Condições de saúde auto-referidas de professores de uma universidade regional. Revista Gaúcha de Enfermagem. Porto Alegre, v. 31, n. 2, p. 270-276, 2010. Disponível em: http://dx.doi.org/10.1590/S1983-14472010000200010. Acesso em: 29 nov 2016.

GUIMARÃES, E. A. A.; ARAÚJO, G. D.; BEZERRA, R.; SILVEIRA, R. C.; OLIVEIRA, V. C. Percepção de técnicos de enfermagem sobre o uso de equipamentos de proteção individual em um serviço de urgência. Ciencia y Enfermería. Concepción, v. 17, n. 3, p. 113-123, 2011. Disponível em: http://dx.doi.org/10.4067/S071795532011000300010. Acesso em: $21 \mathrm{dez} 2016$.

HOEFEL, H. H. K.; LAUTERT, L.; FORTES, C. Riscos ocupacionais no processamento de sistemas de hemodiálise. Revista Eletrônica de Enfermagem. Goiânia, v. 14, n. 2, p. 286-295, 2012. Disponível em: https://www.fen.ufg.br/revista/v14/n2/pdf/v14n2a08.pdf. Acesso em: 20 mar 2017.

LIMA, L. M.; OLIVEIRA, C. C.; RODRIGUES, K. M. R. Exposição ocupacional por material biológico no Hospital Santa Casa de Pelotas - 2004 a 2008. Escola Anna Nery, Revista de Enfermagem. Rio de Janeiro, v. 15, n. 1, p. 96-102, 2011. Disponível em: http://dx.doi.org/10.1590/S1414-81452011000100014. Acesso em: 5 jan 2017.

MACHADO, M. R. M.; MACHADO, F. A. Acidentes com material biológico em trabalhadores de enfermagem do Hospital Geral de Palmas (TO). Revista Brasileira de Saúde Ocupacional. São Paulo, v. 36, n. 124, p. 274-281, 2011. Disponível em: http://dx.doi.org/10.1590/s0303-76572011000200011. Acesso em: 20 dez 2016.

MALAGUTI-TOFFANO, S. E.; SANTOS, C. B.; CANINI, S. R. M. S.; GALVÃO, M. T. G.; BREVIDELLI, M. M.; GIR, E. Adesão às precauções-padrão de profissionais de enfermagem de um hospital universitário. Acta Paulista de Enfermagem. São Paulo, v. 25, n. 3, p. 401-407, 2012. Disponível em: http://dx.doi.org/10.1590/s010321002012000300013. Acesso em: 19 fev 2017.

MARZIALE, M. H.; ZAPPAROLI, A. S.; FELLI, V. E.; ANABUKI, M. H. Rede de Prevenção de Acidentes de Trabalho: uma estratégia de ensino a distância. Revista Brasileira de Enfermagem. Brasília, v. 63, n. 2, p. 250256, 2010. Disponível em: http://dx.doi.org/10.1590/S0034-71672010000200013. Acesso em: 3 dez 2016.

MAURO, M. Y. C.; PAZ, A. F.; MAURO, C. C. C.; PINHEIRO, M. A. S.; SILVA, V. G. Condições de trabalho da enfermagem nas enfermarias de um hospital universitário. Escola Anna Nery, Revista de Enfermagem. Rio de Janeiro, v. 14, n. 2, p. 244-252, 2010. Disponível em: http://dx.doi.org/10.1590/S1414-81452010000200006. Acesso em: 3 dez 2016.

MIRANDA, F. M. A.; STEIN JUNIOR, A. V.; PETRELI, S.; PIRES, M. R.; SOARES, L. G.; RIBEIRAL, B. N.; SARQUIS, L. M. M.; FELLI, V. E. A.; OLIVEIRA, M. C. L. X. Uma contribuição à saúde dos trabalhadores: um guia sobre exposição aos fluídos biológicos. Revista da Escola de Enfermagem da USP. São Paulo, v. 45, n. 4, p. 1018-1022, 2011. Disponível em: http://dx.doi.org/10.1590/S0080-62342011000400033. Acesso em: 6 dez 2016.

MOREIRA, T. R.; ZANDONADE, E.; MACIEL, E. L. N. Risco de infecção tuberculosa em agentes comunitários de saúde. Revista de Saúde Pública. São Paulo, v. 44, n. 2, p. 332-338, 2010. http://dx.doi.org/10.1590/S003489102010000200014. Acesso em: 16 nov 2016.

NASCIMENTO, E. L. A.; VIEIRA, S. B.; CUNHA, T. B. Riscos ocupacionais: das metodologias tradicionais à análise das situações de trabalho. Fractal: Revista de Psicologia. Niterói, v. 22, n. 1, p. 115-126, 2010. Disponível em: http://dx.doi.org/10.1590/S1984-02922010000100009. Acesso em: 22 set 2016.

RIBEIRO, L. C. M.; SOUZA, A. C. S.; NEVES, H. C. C.; MUNARI, D. B.; MEDEIROS, M.; TIPPLE, A. F. V. Influência da exposição a material biológico na adesão ao uso de equipamentos de proteção individual. Ciência, Cuidado \& Saúde. Maringá, v. 9, n. 2, p. 325-332, 2010. Disponível em: https://doi.org/10.4025/cienccuidsaude.v9i2.8282. Acesso em: 2 dez 2016.

RODRIGUES, T. D. F. Fatores estressores para a equipe de enfermagem da unidade de terapia intensiva. Revista Mineira de Enfermagem. Belo Horizonte, v. 16, n. 3, p. 454-462, 2012. Disponível em: http://www.reme.org.br/artigo/detalhes/549. Acesso em: 10 mar 2017. 
RUAS, E. F. G.; SANTOS, L. S.; BARBOSA, D. A.; BELASCO, A. G. S.; BETTENCOURT, A. R. C. Acidentes ocupacionais com materiais perfurocortantes em hospitais de Montes Claros-MG. Revista Mineira de Enfermagem. Belo horizonte, v. 16, n. 3, p. 437-443, 2012. Disponível em: http://www.reme.org.br/artigo/detalhes/547\#. Acesso em: 20 fev 2017.

SANTOS, J. L. G.; VIEIRA, M.; ASSUITI, L. F. C.; GOMES, D.; MEIRELLES, B. H. S.; SANTOS, S. M. A. Risco e vulnerabilidade nas práticas dos profissionais de saúde. Revista Gaúcha de Enfermagem. Porto Alegre, v. 33, n. 2, p. 205-212, 2012. Disponível em: http://dx.doi.org/10.1590/S1983-14472012000200028. Acesso em: 11 mar 2017.

SAPIA, T.; FELLI, V. E. A.; CIAMPONE, M. H. T. Problemas de saúde de trabalhadores de enfermagem em ambulatórios pela exposição a cargas fisiológicas. Acta Paulista de Enfermagem. São Paulo, v. 22, n. 6, p. 808813, 2009. Disponível em: http://dx.doi.org/10.1590/S0103-21002009000600013. Acesso em: 15 nov 2016.

SARQUIS, L. M. M.; FELLI, V. E. A. Os sentimentos vivenciados após exposição ocupacional entre trabalhadores de saúde: fulcro para repensar o trabalho em instituições de saúde. Revista Brasileira de Enfermagem. Brasília, v. 62, n. 5, p. 701-704, 2009. Disponível em: http://dx.doi.org/10.1590/S0034-71672009000500008. Acesso em: 15 nov 2016.

SILVA, E. J.; LIMA, M. G.; MARZIALE, M. H. P. O conceito de risco e os seus efeitos simbólicos nos acidentes com instrumentos perfuro cortantes. Revista Brasileira de Enfermagem. Brasília, v. 65, n. 5, p. 809-814, 2012. Disponível em: http://dx.doi.org/10.1590/S0034-71672012000500014. Acesso em: 15 abr 2017.

SILVA, J. A.; ALMEIDA, A. J.; PAULA, V. S.; VILLAR, L. M. Investigação de acidentes biológicos entre profissionais de saúde. Escola Anna Nery, Revista de Enfermagem. Rio de Janeiro, v. 13, n. 3, p. 508-516, 2009. Disponível em: http://www.scielo.br/pdf/ean/v13n3/v13n3a08.pdf. Acesso em: 15 nov 2016.

SILVINO, Z. R.; MOREIRA, P. S. V.; CHRISTOVAM, B. P.; ESCUDEIRO, C. L.; TEIXEIRA, E. R.; SOUZA, A. M. N. Nursing and work: foundations for health care workers. Revista de Enfermagem UFPE on line. Recife, v. 7, n. esp, p. 4316-4318, 2013. Disponível em: https://doi.org/10.5205/reuol.4134-32743-1-SM-1.0705esp201331. Acesso em: 15 abr 2017.

SULZBACHER, E.; FONTANA, R. T. Concepções da equipe de enfermagem sobre a exposição a riscos físicos e químicos no ambiente hospitalar. Revista Brasileira de Enfermagem. Brasília, v. 66, n. 1, p. 25-30, 2013. Disponível em: http://dx.doi.org/10.1590/S0034-71672013000100004. Acesso em: 9 mai 2017.

VALENÇA, C. N.; AZEVEDO, L. M. N.; OLIVEIRA, A. G.; MEDEIROS, S. S. A.; MALVEIRA, F. A. S.; GERMANO, R. M. The scientific production about occupational health of nursing. Revista de Pesquisa: Cuidado é Fundamental Online. Rio de Janeiro, v. 5, n. 5, p. 52-60, 2013. Disponível em: http://dx.doi.org/10.9789/21755361.2013v5n5esp52. Acesso em: 19 jul 2017.

VALENTE, G. S. C.; CORTEZ, E. A.; ASSIZ, M. M.; OLIVEIRA, J. M.; SANTOS, P. F.; FELICIANO, R. G. Occupational risks and diseases of nursing university lectures: implications to the worker healthy. Revista de Pesquisa: Cuidado é Fundamental Online. Rio de Janeiro, v. 5, n. 1, p. 3267-3275, 2013. Disponível em: http://dx.doi.org/10.9789/2175-5361.rpcfo.v5.1903. Acesso em: 20 jul 2017.

WALL, M. L.; MIRANDA, F. M. A.; SARQUIS, L. M. M.; LABRONICI, L. M.; CRUZ, E. D. A. The beliefs of health workers in occupational accidents with exposure to biological fluid: descriptive research. Online Brazilian Journal of Nursing. Niterói, v. 10, n. 1, p. 1-10, 2011. Disponível em: https://doi.org/10.5935/1676-4285.20113206. Acesso em: 5 jan 2017.

\section{(cc) $\mathrm{EY}$}

Este trabalho está licenciado com uma Licença Creative Commons - Atribuição 4.0 Internacional. 